\title{
LES FACTEURS DE DÉVELOPPEMENT DES BIBLIOTHÈQUES PUBLIQUES QUÉBÉCOISES DEPUIS 1959
}

DEVELOPMENT FACTORS OF THE PUBLIC LIBRARIES IN QUÉBEC SINCE 1959

Dominique Gazo

Candidate au doctorat, ÉBSI, Université de Montréal

Dominique.gazo@umontreal.ca

\section{Résumé}

Cet article est une revue de littérature qui propose de distinguer les différents facteurs de développement des bibliothèques publiques québécoises. La législation, l'action gouvernementale, les modes administratifs, le financement et les normes sont étudiés tout à tour afin de rendre compte du processus de développement des bibliothèques publiques au Québec.

Mots-clés : Bibliothèques publiques - Québec. Action gouvernementale. Modes administratifs, financement et normes.

\section{INTRODUCTION}

Les bibliothèques publiques québécoises ont une histoire relativement courte. Depuis leurs débuts, elles ont accusé un historique si chaotique que la comparaison avec les autres provinces canadiennes a toujours été désastreuse (MITTERMEYER, 1988). Aussi, convient-il de s'interroger sur les différents facteurs particuliers au développement des bibliothèques publiques québécoises.

Postulant que plusieurs facteurs interfèrent et s'interconnectent, il paraît nécessaire de vérifier dans la littérature, quels sont ces facteurs et quelles incidences ils ont pu avoir sur le développement des bibliothèques publiques. Aussi, nous nous proposons ici de dresser une synthèse inédite des faits qui semblent avoir influencé le milieu des bibliothèques publiques du Québec depuis 1959.

\footnotetext{
(c) (1) Esta obra está licenciada sob uma Licença Creative Commons. DOI 10.5007/1518-2924.2009v14nesp1p13
} 


\section{LA LÉGISLATION}

La législation est un des facteurs essentiels du développement des bibliothèques publiques. Le Manifeste de l'UNESCO assure que la bibliothèque publique «doit être soutenue par des textes législatifs spécifiques» (1994). Pour l'IFLA, la législation permet d'assurer la continuité des bibliothèques publiques et leur place dans la structure gouvernementale (2000). Les bibliothèques publiques sont concernées par les textes législatifs concernant leur gestion administrative - gestion du personnel, gestion financière, gestion des actes administratifs - le droit de l'information, et le respect des libertés publiques (GIAPPICONI; CARBONE, 1997). Ce qui nous intéresse ici est la législation sur la gestion propre des bibliothèques publiques. Avant 1960, aucun texte ne légifère en matière de bibliothèque publique au Québec, contrairement aux provinces voisines (CRÉPEAU, 1999; GAGNON, 1985). Comme nous l'avons vu précédemment, Honoré Mercier tente en 1883 de faire adopter une loi semblable à la loi ontarienne sur les bibliothèques publiques, mais retire finalement son projet (GALLICHAN, 1992). Toutefois, sous son mandat, le secrétaire d'État Charles Langelier fait voter une loi permettant aux municipalités de créer des bibliothèques (GALLICHAN, 1992). C'est une loi bien timide dont le contenu confirme en réalité la loi sur les cités et les villes (1870), dans laquelle les municipalités ne sont pas obligées de créer ni de financer une bibliothèque publique (CRÉPEAU, 1999). En 1933, la Commission d'enquête sur la situation des bibliothèques au Canada remarque le sous-développement des bibliothèques québécoises et recommande entre autre de légiférer. La Commission royale d'enquête sur les problèmes constitutionnels formée à la demande Maurice Duplessis, en vient aux mêmes conclusions (CRÉPEAU, 1999). Mais de 1936 à 1939, puis après 1944, Maurice Duplessis s'oppose à toute intervention étatique dans le domaine culturel. Il faut attendre sa mort, en septembre 1959, pour qu'un projet de loi sur les bibliothèques publiques réapparaisse à l'Assemblée (CRÉPEAU, 1999).

La loi concernant les bibliothèques publiques est votée le 7 décembre 1959. Cette loi est assez générale pour «permettre aux administrateurs d'appliquer des solutions pratiques et souvent différentes à chaque phase de développement d'un réseau de bibliothèques publiques »(GAGNON, 1985, p.10). Pour la Commission d'étude sur les bibliothèques publiques, la loi est surtout conçue pour répondre à l'urgence (1987). La loi de 1959 reconnaît le rôle primordial des bibliothèques publiques et crée une Direction des bibliothèques publiques comprenant la Commission des bibliothèques publiques et le Service 
des bibliothèques publiques (COMMISSION D'ÉTUDE SUR LES BIBLIOTHÈQUES PUBLIQUES DU QUÉBEC, 1987; CRÉPEAU, 1999; GAGNON, 1985), mais elle reste muette sur le financement (CRÉPEAU, 1999).

Dans les années 1980, la Direction des bibliothèques publiques est démantelée. Or, 12 articles sur les 15 que contenait la loi de 1959 concernaient l'organisation de cette direction. Autant dire que la loi de 1959 n'a plus de raison d'être (CRÉPEAU, 1999). En 1987, la Commission d'étude sur les bibliothèques publiques du Québec émet 14 recommandations (sur 76) concernant une législation nouvelle, s'appuyant sur les recommandations de Gardner (1972). Mais elles restent lettres mortes. La loi de 1959 est abrogée de fait en 1992 par la loi 52, tandis que la loi créant le ministère de la culture est votée (CRÉPEAU, 1999; PANNETON, 1995).

Aujourd'hui, c'est la loi sur le ministère de la culture et des communications de 1994 qui fait office de loi pour les bibliothèques publiques (QUÉBEC. MINISTÈRE DE LA CULTURE ET DES COMMUNICATIONS, 2004). En réalité, cette loi est consacrée à l'organisation du nouveau ministère. Un chapitre, le troisième, est consacré aux bibliothèques publiques et CRSBP, comprenant 7 alinéas :

- le ministre consulte les municipalités et les milieux professionnels pour définir les besoins et établir les objectifs des services offerts par les bibliothèques publiques;

- le ministre établit les priorités et les moyens pour promouvoir la coopération des services, fournir un appui professionnel ou technique et recueillir et publier les statistiques;

- le ministre peut autoriser la création d'un CRSBP;

- un CRSBP peut demander à changer de nom, réaliser une fusion, demander des lettres patentes ou sa dissolution;

- en cas de dissolution d'un CRSBP, le ministre désigne la municipalité, la régie intermunicipale ou le CRSBP qui héritera de ses biens;

- un CRSBP doit transmettre au ministre un rapport d'activité;

- le ministre exerce les mêmes fonctions pour les CRSBP et les bibliothèques publiques. Le manque de législation propre aux bibliothèques publiques est unanimement décrié (ASSOCIATION POUR L'AVANCEMENT DES SCIENCES ET DES TECHNIQUES DE LA DOCUMENTATION, 1998; COMMISSION D’ÉTUDE SUR LES BIBLIOTHÈQUES PUBLIQUES DU QUÉBEC, 1987); les 7 alinéas de la loi sur le ministère de la culture et des communications ne constituant pas un cadre législatif suffisant (CRÉPEAU, 1999; SÉGUIN, 
1995). Pour Séguin, les conséquences sont graves : la non-reconnaissance des bibliothèques publiques comme service essentiel dans une société dite d'information, la possibilité de tarifer les services, l'absence de normes et de critères d'excellence qui assureraient le développement et le financement des bibliothèques publiques - le guide de l'Association pour l'avancement des sciences et des techniques de la documentation (ASTED) qui fait office de normes au Québec a paru un an après l'article de Séguin -, le manque de vision globale pour un développement harmonieux des bibliothèques publiques québécoises (1991). Séguin écrit: «Nul besoin d'être grand clerc pour comprendre que le laxisme actuel constitue un obstacle rédhibitoire au développement d'un réseau moderne de bibliothèques publiques et contribue à pérenniser les inégalités d'accès à l'information » (1995, p.11).

L'ASTED a bien émis quelques recommandations concernant la nécessité d'une loi spécifique aux bibliothèques publiques dans son mémoire (1991) soumis à la Commission de la culture qui étudiait les recommandations du rapport Une politique de la culture et des arts et son mémoire soumis au gouvernement lors du sommet sur la lecture et le livre en 1998 (1998), mais là encore elles n'ont pas été suivies.

La loi portant réforme de l'organisation territoriale municipale des régions métropolitaines de Montréal, de Québec et de l'Outaouais (Loi 170), votée le 20 décembre 2000, imposait à certaines communes de fusionner à compter du 1er janvier 2002. Ce fut chose faite, mais rien dans la loi ne permettait d'assurer l'avenir des bibliothèques dans ces nouvelles villes (LEGAULT, 2001). Les bibliothèques des communes fusionnées ont alors dû constituer des réseaux. Cette intégration « est le passage obligé vers des services de meilleure qualité et à moindre coût » (SÉGUIN, 2001, p.2), à condition de niveler par le haut (LEGAULT, 2001). Mais elle n'est pas chose aisée. Le cas de Montréal par exemple démontre une grande disparité entre les bibliothèques publiques des villes fusionnées. Il faut donc considérer le financement, les conditions des employés, le développement d'une nouvelle structure organisationnelle, l'harmonisation des différents services, l'homogénéisation des politiques et procédures, des technologies, des services techniques et financiers (LEGAULT, 2001). Legault préconise de suivre les exemples de Toronto et Ottawa. En Ontario, une liste de contrôle avait été établie en 1998 par le Service des bibliothèques de l'Ontario pour pointer les domaines à étudier lors de la fusion des bibliothèques publiques (LEGAULT, 2001). Elle comprenait la gestion et la gouvernance, les communications, la direction générale, les ressources humaines, les finances et l'administration, les installations, l'accessibilité aux collections, les services de livraison, le plan de service, le développement des collections. 
Toutefois, à la faveur d'un renversement de majorité, la fusion a été remise en cause et le démembrement des communes rendu possible à compter du $1^{\text {er }}$ janvier 2006 par la loi concernant la consultation des citoyens sur la réorganisation territoriale de certaines municipalités (Projet de loi $\mathrm{n}^{\circ}$ 9, sanctionné le 18 décembre 2003). Les résultats des référendums sont éloquents, notamment à Montréal et à Longueuil : 15 villes démembrées sur 28 à Montréal et 4 sur 7 à Longueuil.

La revue de littérature montre l'unanimité face à la nécessité d'une loi spécifique aux bibliothèques publiques au Québec; une loi qui montrerait la place de la bibliothèque publique dans la société de l'information, qui établirait les responsabilités de chaque pilier de gouvernement, qui créerait un service central destiné à promouvoir et organiser le développement des bibliothèques publiques, qui imposerait la gratuité des services de base et qui assurerait des sources de financement. L'absence de loi réelle, et la confusion née des fusions suivies ou non des démembrements, témoignent de la fragilité des bibliothèques publiques face aux pouvoirs politiques. Une loi semble bien être un facteur primordial du développement des bibliothèques publiques. Même si la loi ne règle pas tous les problèmes (GARDNER, 1972), elle reste un phare dans un océan d'incertitude.

\section{L'ACTION GOUVERNEMENTALE}

L'action gouvernementale est ici entendue au sens de choix politiques pour le développement des bibliothèques publiques québécoises. Panneton distingue deux grandes périodes dans l'évolution des bibliothèques publiques depuis 1959 (1995). La première comprend, selon ses termes, le coup d'envoi de 1959 à 1973, l'accessibilité au livre et l'harmonisation des programmes de 1973 à 1979, et le plan Vaugeois de 1980 à 1985. La seconde période, de 1986 à 1995, est pour Panneton, une période de rupture dans la politique de l'État concernant les bibliothèques publiques (1995).

C'est l'adoption de la loi sur les bibliothèques publiques en 1959 qui a donné le coup d'envoi des bibliothèques publiques au Québec (PANNETON, 1995). Cette loi crée la Direction des bibliothèques publiques, constituée de deux organismes chargés d'aider à leur développement : le Service des bibliothèques publiques, et la Commission des bibliothèques publiques. Le Service veille à l'application des programmes, à la distribution des subventions 
gouvernementales, et à la coopération avec les municipalités, les associations de bibliothécaires et les autres groupes intéressés au développement des bibliothèques publiques (PANNETON, 1995). Le Service est aussi un organisme chargé de faire la promotion des bibliothèques publiques. Il constitue un centre de documentation spécialisé. Il publie également des statistiques annuelles (GAGNON, 1985). La Commission est un organisme consultatif chargé de conseiller le ministre en ce qui concerne les bibliothèques publiques (GAGNON, 1985; PANNETON, 1995). En 1961, la Commission des bibliothèques publiques mène une enquête "pour connaître la situation des bibliothèques, pour évaluer leur fonctionnement, leur rendement et l'intérêt des autorités municipales à leur égard» (COMMISSION D’ÉTUDE SUR LES BIBLIOTHÈQUES PUBLIQUES DU QUÉBEC, 1987, p.11). Les résultats montrent leur sous-développement (COMMISSION D’ÉTUDE SUR LES BIBLIOTHÈQUES PUBLIQUES DU QUÉBEC, 1987; GAGNON, 1985; PANNETON, 1995). L'absence d'une loi sur les bibliothèques, l'indifférence des conseils municipaux concernant la lecture publique, la pénurie de bibliothécaires et une population faiblement scolarisée et imprégnée de préjugés envers la lecture, sont autant de facteurs qui expliqueraient cet état de sous-développement (COMMISSION D’ÉTUDE SUR LES BIBLIOTHÈQUES PUBLIQUES DU QUÉBEC, 1987; GAGNON, 1985). Le Ministère décide alors de sensibiliser les municipalités au fait des bibliothèques publiques autonomes, et de les soutenir financièrement et techniquement (PANNETON, 1995). Une autre enquête de la Commission en 1962 démontre que toute la population québécoise n'est pas desservie par une bibliothèque publique, notamment dans les régions les plus reculées des grands centres urbains. Le Ministère décide alors de mettre en place un réseau de bibliothèques publiques pour les régions rurales, par le biais de bibliothèques centrales de prêt, $\mathrm{BCP}$ (PANNETON, 1995). Le territoire est découpé en 21 régions. La première BCP voit le jour en Mauricie en 1961, puis en Outaouais en 1964 et au Saguenay-Lac-Saint-Jean en 1972 (GAGNON, 1985). La Direction des bibliothèques publiques s'inspire également de diverses études étrangères sur leur législation concernant les bibliothèques publiques, l'organisation et le fonctionnement de leurs services régionaux, les modes de distribution des subventions, les types de catalogage et de classification, les bibliobus, la coopération (GAGNON, 1985). Des programmes d'aide financière sont mis en place, basés sur le nombre d'habitants dans les municipalités, leur budget, et la qualité du personnel professionnel (GAGNON, 1985). Ils visent « l'augmentation de la contribution municipale, l'implantation de nouvelles bibliothèques, le recrutement de personnel qualifié, l'amélioration de la qualité des services offerts et la 
municipalisation des bibliothèques privées ou d'associations » (PANNETON, 1995, p.154). Plusieurs bibliothèques d'associations sont ainsi municipalisées (GAGNON, 1985). Toutefois, ce coup d'envoi paraît plus être une période d'expérimentation et d'acquisition de connaissances que de progrès réels pour le développement des bibliothèques publiques (COMMISSION D’ÉTUDE SUR LES BIBLIOTHÈQUES PUBLIQUES DU QUÉBEC, 1987). Pour Gagnon, les causes de cette lenteur sont les autres priorités budgétaires, l'attitude passive de la population principalement rurale, «d'un niveau de scolarité peu élevé et imprégnée de préjugés défavorables à la lecture », et le manque d'intérêt de l'État et des municipalités pour investir dans ce secteur (1985, p.9-10). Mais, à la défense du ministère, Gagnon reconnaît que l'État n'avait pour seul soutien que les associations de bibliothécaires, la Direction des bibliothèques publiques et quelques intellectuels. L'État avait aussi d'autres priorités: la démocratisation scolaire et l'universalisation des programmes de santé (GAGNON, 1985).

Les auteurs s'entendent pour dire qu'il faut attendre les années 1970 pour qu'une politique plus incitative soit adoptée (GAGNON, 1985; PANNETON, 1995; COMMISSION D’ÉTUDE SUR LES BIBLIOTHÈQUES PUBLIQUES DU QUÉBEC, 1987). L'introduction d'une nouvelle gestion gouvernementale par programme se décline pour la Direction des bibliothèques publiques dans le programme de « développement d'un réseau de bibliothèques publiques »(GAGNON, 1985, p.15). Le Conseil du trésor demande des mémoires aux différents ministères et services pour justifier leurs rôles et activités. Le document du Service des bibliothèques publiques est présenté au Conseil du trésor en 1973. Il démontre la nécessité d'une action gouvernementale, détermine les objectifs, et établit un plan de développement pour 1975-1990. Le document est accepté, ce qui signifie que l'État s'engage à suivre les recommandations de ce mémoire (GAGNON, 1985). Des mesures plus formelles sont ainsi mises en place durant cette période, qui permettent une plus grande coordination des politiques : une réglementation progressive du marché du livre; la publication d'un cahier de normes qualitatives et quantitatives permettant de mesurer les conditions d'efficacité des bibliothèques; la publication d'un bulletin d'information et de statistiques trimestriel BiblioContact; la tenue d'une campagne de promotion de la lecture (COMMISSION D'ÉTUDE SUR LES BIBLIOTHÈQUES PUBLIQUES DU QUÉBEC, 1987; PANNETON, 1995). Le Livre blanc sur le développement culturel publié en 1978 appuie l'élaboration d'une politique de la lecture (COMMISSION D’ÉTUDE SUR LES BIBLIOTHÈQUES PUBLIQUES DU QUÉBEC, 1987). La coopération est facilitée par un effort de concertation et de 
rationalisation pour la mise en commun et l'utilisation maximale des ressources, une coordination accrue entre bibliothèques municipales et scolaires, l'utilisation collective des services techniques de la Centrale des bibliothèques, la création de bibliothèques intermunicipales (PANNETON, 1995). Le calcul des subventions est modifié. Il est alors basé sur l'évaluation foncière des municipalités dans un souci de péréquation (GAGNON, 1985; PANNETON, 1995). De nouveaux programmes d'aide sont offerts aux municipalités, instaurant des conditions d'admissibilité afin de s'assurer d'une contribution municipale minimale et d'améliorer la qualité des services offerts par les bibliothèques en précisant leurs objectifs, moyens et indicateurs de performance (GAGNON, 1985). Il s'agit d'un programme d'aide à la création d'une bibliothèque publique autonome (sur 2 ans), d'un programme d'aide pour les 3 premières années d'emploi du bibliothécaire professionnel, et d'un programme expérimental (1978-1981) d'aide à l'animation des bibliothèques publiques autonomes (GAGNON, 1985). Les rencontres avec les associations des bibliothécaires et les études caractérisent également cette période (COMMISSION D’ÉTUDE SUR LES BIBLIOTHÈQUES PUBLIQUES DU QUÉBEC, 1987). Le réseau des BCP n'est pas terminé. Le découpage du territoire est réduit à 10 régions au lieu des 21 prévues initialement (GAGNON, 1985). Le réseau est complété en 1981. Le Service des bibliothèques publiques nomme un coordonnateur du réseau pour étudier les revendications des $\mathrm{BCP}$ et assurer la liaison entre le Service et les BCP (GAGNON, 1985). Le Service des bibliothèques publiques reçoit en 1978 le mandat de préparer un plan quinquennal, eu égard aux résultats des bibliothèques publiques québécoises comparés à ceux de l'Ontario et du Danemark (GAGNON, 1985). La gestion par programme aboutit pour la Commission des bibliothèques publiques à un dégagement de l'administration des subventions (COMMISSION D'ÉTUDE SUR LES BIBLIOTHÈQUES PUBLIQUES DU QUÉBEC, 1987; GAGNON, 1985). Pour la première fois, les bibliothèques publiques font l'objet d'un atelier lors du Congrès de l'Union des municipalités du Québec en 1979 (GAGNON, 1985). L’État incite les municipalités à participer au développement des bibliothèques publiques et se dote d'outils d'évaluation et de coopération des services (COMMISSION D’ÉTUDE SUR LES BIBLIOTHÈQUES PUBLIQUES DU QUÉBEC, 1987).

Les études sur la situation des bibliothèques publiques, la publication du Livre blanc (1978) sur le développement culturel et la pression des associations de bibliothécaires, incitent le ministère à intervenir rapidement dans le développement des bibliothèques (GAGNON, 1985). En 1980, le ministre des Affaires culturelles, Denis Vaugeois, présente un plan 
quinquennal de développement des bibliothèques publiques qui prévoit de nouvelles mesures pour inciter les municipalités à investir davantage, de compléter l'aménagement du territoire en matière de bibliothèque publique et le réaménagement des bibliothèques existantes (COMMISSION D’ÉTUDE SUR LES BIBLIOTHÈQUES PUBLIQUES DU QUÉBEC, 1987; PANNETON, 1995). Le programme fait l'objet d'une campagne de promotion destinée à informer les municipalités. Le programme d'aide à la rénovation et à la construction des bibliothèques donne lieu à la multiplication et au rajeunissement des bibliothèques publiques au Québec durant cette période (PANNETON, 1995). Le taux de l'aide varie en fonction du niveau de conformité aux normes (GAGNON, 1985). Mais le programme d'aide à la création de bibliothèques municipales est rapidement abandonné (GAGNON, 1985). Le programme d'aide au fonctionnement est renforcé puisqu'il prévoit une compensation pour la perte financière engendrée par la loi sur le développement des entreprises québécoises dans le domaine du livre (Loi 51) qui oblige les bibliothèques à acquérir leurs livres dans les librairies agréées. Il comprend une subvention égale à $50 \%$ du coût des livres achetés et une subvention de base variant en fonction des dépenses de fonctionnement (hormis les acquisitions de livres), en fonction de la part consentie par la municipalité (GAGNON, 1985). Les règles de calcul pour les subventions sont simplifiées, les conditions d'admissibilité des municipalités aux subventions sont éliminées (GAGNON, 1985). Ce faisant, pour Laborit et Dubois, le ministère perd ce qui avait «jusque-là assuré une garantie minimale d'un développement harmonieux des bibliothèques publiques » notamment l'emploi d'un bibliothécaire professionnel et la gratuité des services (1983, p.73). Gagnon (1985) parle d'une nouvelle orientation, en ce sens que la gestion des programmes est désormais déconcentrée, décloisonnant du même coup les services gouvernementaux. Aussi, en 1983, le Service des bibliothèques publiques est-il démembré (GAGNON, 1985). Le secteur des bibliothèques publiques se retrouve avec les secteurs des arts et des établissements culturels dans un Service des programmes régionaux au sein du Sous-ministère au développement régional (GAGNON, 1985). Par ce plan, l'État démontre pour la première fois, que les bibliothèques publiques sont une priorité, dans son discours sur le budget 1980 (GAGNON, 1985). Ce plan suscite l'intérêt des élus municipaux, et ce faisant, change les attitudes et mentalités de ceux-ci vis-à-vis de la lecture publique (LABORIT; DUBOIS, 1983). Mais, c'est aussi durant cette période que le Service des bibliothèques publiques disparaît, que le bulletin d'information Biblio-Contact n'est plus édité, que le centre de documentation est intégré à celui du ministère et perd de sa spécialité, que la Commission des bibliothèques publiques perd de son influence au sein du 
ministère (COMMISSION D'ÉTUDE SUR LES BIBLIOTHÈQUES PUBLIQUES DU QUÉBEC, 1987; GAGNON, 1985, MITTERMEYER, 1988).

Une fois le plan Quinquennal terminé, l'action gouvernementale change radicalement; ce qui fait dire à Savard qu' "à cette période d'euphorie succéda des lendemains qui déchantent » (1998, p.144). Suite à la réorganisation ou la disparition des organismes et outils qui organisaient le secteur des bibliothèques publiques au sein du ministère des Affaires culturelles, celui-ci se doit de redéfinir sa politique après le plan Vaugeois (PANNETON, 1995). Les résultats obtenus en 1985 démontrent que les objectifs fixés dans le plan quinquennal de 1979 ont été partiellement atteints et ont remis en cause l'action gouvernementale en matière de lecture publique (COMMISSION D'ÉTUDE SUR LES BIBLIOTHÈQUES PUBLIQUES DU QUÉBEC, 1987). La déception est grande en effet quand le gouvernement coupe de $25 \%$ les subventions d'aide au fonctionnement des bibliothèques et impose un moratoire sur le programme d'aide à la construction et à la rénovation pour des raisons budgétaires (PANNETON, 1995). Aussi, la ministre Lise Bacon, instaure-t-elle en 1986-1987 une commission d'étude sur les bibliothèques publiques ayant pour fonction de :

\footnotetext{
faire le point sur l'état de la situation des bibliothèques publiques du Québec et proposer des orientations pouvant guider l'action de l'État dans ce domaine (...) quant aux moyens à prendre pour poursuivre, sur une période de cinq années, le développement des bibliothèques publiques; quant au partage des responsabilités entre l'État et les municipalités relativement à ce développement; quant aux modes de financement de ce développement (COMMISSION D'ÉTUDE SUR LES BIBLIOTHĖQUES PUBLIQUES DU QUÉBEC, 1987, p.4).
}

Cette commission, présidée par Philippe Sauvageau, reçoit 101 mémoires, et formule 76 recommandations dont quelques-unes sont suivies d'effets: la révision de la Loi sur les bibliothèques publiques mais le projet de loi avorte, la création d'une Direction générale des bibliothèques publiques en 1989, la mise à jour des normes pour les bibliothèques publiques mais le projet n'abouti pas, l'augmentation de la participation financière du MAC, l'aide financière à l'informatisation des $\mathrm{BCP}$, la détermination d'une stratégie d'informatisation des bibliothèques autonomes, l'appui aux regroupements régionaux de bibliothèques publiques, l'élaboration de critères d'évaluation et la levée du moratoire sur les équipements culturels (PANNETON, 1995, p.156).

L'aide financière aux bibliothèques autonomes ne porte plus guère que sur l'enrichissement des collections, et le fonctionnement des services. Pour les BCP, l'aide financière porte sur la 
construction et la rénovation. En 1992, les BCP deviennent les Centres Régionaux de Services aux Bibliothèques Publiques, CRSBP (PANNETON, 1995).

La politique des arts et de la culture du Québec, en 1992, laisse peu de place aux bibliothèques publiques. La création du Ministère de la culture en 1992 (Loi 52), puis celle du Ministère de la culture et des communications en 1994 démontrent le désengagement de l'État: les municipalités sont les maîtres d'œuvre du développement des bibliothèques publiques et la Direction du livre, de la lecture et des bibliothèques publiques créée en 1989 est abolie et ses activités décentralisées (PANNETON, 1995).

La politique de la lecture et du livre de 1998 vise à augmenter les ressources documentaires, humaines, l'accessibilité et les équipements de manière à rattraper le retard des bibliothèques publiques québécoises et à promouvoir la coopération des services (QUÉBEC (PROVINCE), 1998, p.31). L'accent est mis sur l'enrichissement des collections, avec un soutien particulier aux documents québécois et aux documents acquis pour compléter les collections des bibliothèques scolaires. Les règles d'attribution des subventions sont assouplies et l'enveloppe budgétaire élargie pour les bibliothèques publiques autonomes et les CRSBP. Le ministère s'engage également à soutenir financièrement la formation continue du personnel professionnel et technique à la demande des associations de bibliothécaires (QUÉBEC (PROVINCE), 1998, p.36). Les projets des municipalités sans bibliothèque, les projets de rénovation des bibliothèques pour atteindre les normes, les projets polyvalents de type maison de la culture, les projets inter-municipaux, les projets qui allient bibliothèques municipales et bibliothèques scolaires sont privilégiés par la nouvelle politique de la lecture et du livre. Enfin, le ministère veut élargir l'accès au livre adapté pour les personnes handicapées sur tout le territoire québécois, par l'intermédiaire de la Grande Bibliothèque (QUÉBEC (PROVINCE), 1998). Afin d'accentuer la mise en commun des ressources documentaires, le ministère s'engage à aider financièrement les bibliothèques dans leurs projets d'informatisation d'ici 2001, leurs projets de compatibilité des systèmes afin de constituer un réseau, et les bibliothèques qui agissent comme des centres-ressources par le biais du prêt entre bibliothèques, notamment les CRSBP. Le projet de Grande Bibliothèque répond également à cette exigence de coopération et de mise en place d'un réseau des bibliothèques publiques québécoises (QUÉBEC (PROVINCE), 1998). Dans cette politique, la construction et la rénovation des bibliothèques ne sont pas mentionnées puisqu'elles font partie du programme général de soutien aux équipements culturels. Encore une fois, l'accent de l'État est mis sur les collections, tandis que « la plus grande lacune des bibliothèques québécoises 
(...) est son manque chronique de ressources humaines » (SAVARD, 1998, p.155). Actuellement, les aides financières offertes aux bibliothèques publiques concernent le soutien au développement des collections des bibliothèques publiques autonomes et le soutien aux CRSBP. Le programme de soutien aux équipements culturels concernant les bibliothèques publiques autonomes, les bibliothèques affiliées aux CRSBP, et les CRSBP. Quatre types de projets sont admissibles à ce programme : l'achat et l'installation d'équipement et/ou de mobilier spécialisé; l'achat d'un immeuble; la réalisation des travaux de construction, d'agrandissement, de rénovation, d'aménagement; et le maintien des actifs que sont les bâtiments, les équipements et le mobilier (QUÉBEC (PROVINCE). MINISTÈRE DE LA CULTURE ET DES COMMUNICATIONS, 2004). Le programme de soutien au développement des collections des bibliothèques publiques autonomes vise :

\footnotetext{
à favoriser la gratuité des services de base des bibliothèques publiques de manière à en faciliter l'accès et à accroître leur clientèle; à favoriser la diffusion de la culture et de la littérature québécoise en encourageant l'achat de livres et de périodiques édités au Québec (QUÉBEC (PROVINCE). MINISTÈRE DE LA CULTURE ET DES COMMUNICATIONS, 2004).
}

La revue de littérature concernant l'action gouvernementale pour le développement des bibliothèques publiques québécoises démontre un manque de constance. Aucune des actions entreprises ne s'est inscrite dans la durée, comme l'indique le titre d'un article de Mittermeyer, Le développement des bibliothèques publiques du Québec : un pas en avant, deux pas en arrière (1988). Or, l'action gouvernementale a un impact important sur le développement des bibliothèques publiques; chaque étape vue ci-dessus l'a démontré à sa manière. Aussi, le désintéressement de l'État est-il à craindre. L'action gouvernementale depuis 1995 est restée sensiblement la même, en ce sens qu'il n'existe toujours pas de direction des bibliothèques publiques, les normes n'ont pas été remises à jour depuis 1996, et la loi de 1994 sur le Ministère de la culture et des communications fait toujours office de loi sur les bibliothèques publiques. La politique du livre et de la lecture de 1998 n'a pas effacé la crainte du désintéressement de l'État puisque son action est très partielle. Une action d'envergure et inscrite dans la durée fait semble-t-il défaut au développement des bibliothèques publiques québécoises.

\section{LES MODES ADMINISTRATIFS}


Deux modes administratifs existent dans le monde des bibliothèques : la tutelle municipale et la gestion par un conseil d'administration. Au Québec, on trouve principalement des bibliothèques sous tutelle municipale. Cependant, quelques conseils d'administration existent. Pour la Commission d'étude sur les bibliothèques publiques du Québec, «les modes organisationnels peuvent, en eux-mêmes favoriser la performance ou constituer une entrave à l'excellence » (1987, p.114). Certains ont eu vite fait de reprocher à la structure administrative québécoise le fameux retard des bibliothèques du Québec comparées à leurs voisines ontariennes ou américaines. Mais la Commission a dû convenir que les études consultées sur ce point n'ont pas permis de conclure par la négative ni l'affirmative (1987). Le débat sur les modes administratifs n'a pas abouti à un éclairage satisfaisant (Mittermeyer, doc. 44, 1986).

Dans le monde anglo-saxon, les bibliothèques publiques ont été confiées à des conseils d'administration autonomes et indépendants des municipalités. Dans le monde latin, et québécois, la tendance est à la municipalisation des bibliothèques publiques (COMMISSION D’ÉTUDE SUR LES BIBLIOTHÈQUES PUBLIQUES DU QUÉBEC, 1987). Les raisons du choix du mode de gestion municipale sont dues aux données démographiques particulières (très petites municipalités), au «tempérament individualiste de ces latins que sont les Québécois », à la réticence des élus pour déléguer leurs responsabilités, et au fonctionnement des subventions gouvernementales pour les municipalités concernant les bibliothèques publiques (MATTE, 1987, p.47). Toutefois, l'ASTED ouvre la porte au changement puisqu'elle permet aux conseils municipaux de confier la responsabilité des bibliothèques publiques à une corporation (1996).

La tutelle municipale signifie que les décisions sont prises par le conseil municipal, notamment le vote du budget, et que le personnel est constitué de fonctionnaires municipaux (COMMISSION D’ÉTUDE SUR LES BIBLIOTHÈQUES PUBLIQUES DU QUÉBEC, 1987). Les principales faiblesses de ce mode administratif sont finalement la place de la bibliothèque au sein du conseil municipal parmi les nombreux autres services aux citoyens, et la hiérarchisation des rapports entre les professionnels et les élus municipaux (MITTERMEYER, 1987).

Les fonctions des conseils d'administration sont :

de représenter les intérêts du public dans les domaines de la lecture et de la documentation gratuites; de voir au contrôle des biens et services des bibliothèques publiques; de formuler des «politiques » administratives ou de supporter celles qui sont élaborées par les professionnels de la bibliothéconomie; d'isoler, en quelque sorte, la bibliothèque publique du gouvernement local; d'assurer par leurs démarches 
auprès des entreprises et même des pouvoirs publics un support financier adéquat pour les bibliothèques publiques (MATTE, 1987, p.46).

Les qualités de ce mode de gestion sont leur force de conviction, les connaissances et le dynamisme des membres des conseils d'administration, leur capacité à s'adapter aux différentes situations (MATTE, 1987; MITTERMEYER, 1987). Le conseil est, entre autres, un organe de relations publiques (CHRISTENSON, 1995; MITTERMEYER, 1986). Le principal inconvénient de ce mode administratif, aux dires des municipalités, est son autonomie qui constitue un obstacle à la planification budgétaire municipale (MITTERMEYER, 1987). Selon les professionnels, le problème de ce mode de gestion, est de sensibiliser les membres au fur et à mesure de leur élection au sein du conseil d'administration et de gérer les différents intérêts de chacun de ces membres (MITTERMEYER, 1987)

Si les études n'ont pas réussi à démontrer l'influence bénéfique des conseils d'administration sur le développement des bibliothèques publiques, il reste que la question est toujours en suspens. Toutefois, changer de mode de gestion ne va pas de soi. Il n'y a pas de solution modèle, mais sans doute une adaptation au contexte de chaque bibliothèque.

\section{LE FINANCEMENT}

Le financement est assuré par les subventions du ministère, les contributions municipales et autres revenus. Il s'agit ici de voir l'évolution du financement global depuis 1959, les aides gouvernementales disponibles, la part des contributions gouvernementales et municipales, et finalement la tarification des services comme autre revenu.

Globalement, le financement est à la hausse depuis le début des bibliothèques publiques. Par exemple, « en dollars constants de 1981, les bibliothèques publiques du Québec ont plus que septuplé leurs effort financier en 1989 par rapport à $1961 »$ (BAILLARGEON, 1992, p.141); les dépenses ont augmenté de près de 60\% entre 1960 et 1995 (SAVARD, 1998, p.149). La période la plus profitable aux bibliothèques publiques s'est étendue de 1977 à 1985 (MORIN; LAJEUNESSE, 1996, p.24). Cet effort financier a particulièrement contribué à élargir la population desservie et le volume de prêt de documents (BAILLARGEON, 1992, p.141), mais n'a pas permis de rattraper le retard du Québec par rapport à l'Ontario en termes 
financiers et en termes de résultats (SAVARD, 1998). Deux facteurs pourraient expliquer la contre-performance québécoise : l'augmentation de la tarification des services de base des bibliothèques publiques et l'insuffisance de ressources humaines compétentes (SAVARD, 1998), deux facteurs qui découlent directement du financement des bibliothèques.

Nous avons vu plus haut les fluctuations des actions gouvernementales qui ont eu des incidences sur les aides financières gouvernementales pour les bibliothèques publiques. Actuellement, celles-ci sont de trois ordres : le soutien au développement des collections des bibliothèques publiques autonomes, le soutien aux centres régionaux de services aux bibliothèques publiques et le soutien aux équipements culturels. Les aides financières de l'État concernent donc principalement l'accroissement des collections et la mise aux normes des bâtiments, tandis que les bibliothèques publiques manquent en premier lieu de ressources humaines compétentes (SAVARD, 1998). Aucune mesure incitative, ni coercitive, n'incite les municipalités à investir en ce sens (SAVARD, 1998).

La part des contributions gouvernementales et municipales a beaucoup varié depuis le début des bibliothèques publiques au Québec. La Commission d'étude sur les bibliothèques publiques a retracé l'historique de ces variations jusqu'en 1986. Elle a montré que les subventions de l'État pour les bibliothèques publiques ont véritablement augmenté dans les années 1970 (1987). Mais c'est le plan Vaugeois qui a offert un envol des subventions_une augmentation de près de 150\%_(SAVARD, 1998, p.144), aussitôt suivi par la remise en question de l'aide financière gouvernementale dans la seconde moitié des années 1980 (COMMISSION D’ÉTUDE SUR LES BIBLIOTHÈQUES PUBLIQUES DU QUÉBEC, 1987; MORIN; LAJEUNESSE, 1996; SAVARD, 1998). Le rapport entre la part des contributions financières de l'État et des municipalités s'est inversé à la suite du plan Vaugeois, lequel a eu un impact déterminant sur la croissance financière des municipalités au fonctionnement des bibliothèques (CHAREST, 1995; COMMISSION D’ÉTUDE SUR LES BIBLIOTHÈQUES PUBLIQUES DU QUÉBEC, 1987; SÉGUIN, 1995). À titre d'exemple, en 1986, 82,5\% des revenus des bibliothèques publiques autonomes provenaient des municipalités, 13,8\% de l'État et 3,7\% étaient des revenus d'appoint tels que les amendes et la tarification des services (COMMISSION D'ÉTUDE SUR LES BIBLIOTHÈQUES PUBLIQUES DU QUÉBEC, 1987, p. 45). Les municipalités ont donc assumé leurs responsabilités, « au-delà de l'idée généralement répandue » puisque leur effort financier pour les bibliothèques publiques dépassait l'augmentation du coût de la vie (MORIN; LAJEUNESSE, 1996, p.25). La progression des contributions municipales était fulgurante 
depuis le début des années 1960 (SAVARD, 1998, p.143), même si l'effort financier des municipalités s'est affaibli après 1985 (MORIN; LAJEUNESSE, 1996, p.26). On remarque également un écart conséquent entre l'effort financier des communes anglophones et des communes francophones (SAVARD, 1998). Si les contributions municipales ont augmenté, il n'empêche qu'elles se situaient en bas de la moyenne canadienne (COMMISSION D'ÉTUDE SUR LES BIBLIOTHÈQUES PUBLIQUES DU QUÉBEC, 1987, p.52). Le budget ministériel pour les bibliothèques publiques, lui, a chuté de 12\% entre 1987 et 1995 (SAVARD, 1998, p.149). Charest écrit :

\footnotetext{
En réduisant à la portion congrue les subventions, le gouvernement met en sérieux danger de dislocation le réseau des bibliothèques publiques. Il crée un déséquilibre entre les municipalités qui pourront continuer leurs efforts et celles qui ne le pourront pas, fautes de moyens financiers ou de volonté politique devant le gâchis du gouvernement (1995, p.15).
}

Depuis 1987, la situation a continué d'évoluer dans le même sens : la part de l'État baisse, tandis que celle des municipalités augmente légèrement ou stagne et celle des revenus connexes augmente, que ce soit pour les bibliothèques publiques autonomes ou pour les CRSBP (QUÉBEC (PROVINCE). MINISTÈRE DE LA CULTURE, 1993; QUÉBEC (PROVINCE). MINISTÈRE DE LA CULTURE ET DES COMMUNICATIONS, 1994-2005; QUÉBEC (PROVINCE). MINISTÈRE DE LA CULTURE, DES COMMUNICATIONS ET DE LA CONDITION FÉMININE, 2007; QUÉBEC (PROVINCE). MINISTÈRE DES AFFAIRES CULTURELLES, 1987-1992).

L'UNESCO et l'IFLA et autres associations professionnelles comme l'ALA se sont prononcées en faveur de la gratuité des bibliothèques publiques (INTERNATIONAL FEDERATION OF LIBRARY ASSOCIATIONS AND INSTITUTIONS, 2000; LAVOIE, 2000; ROUSSEAU, 1990). L'IFLA prend même la peine de spécifier dans ses principes directeurs que les usagers ne peuvent être mis à contribution sous peine de réduire l'accès aux bibliothèques et par-là même bafouer le principe fondateur de la bibliothèque publique, celui de l'accès pour tous (INTERNATIONAL FEDERATION OF LIBRARY ASSOCIATIONS AND INSTITUTIONS, 2000). Rappelons également que la nouvelle politique du livre offre une bonification pour les bibliothèques gratuites (QUÉBEC (PROVINCE), 1998). Pourtant, la part des revenus autres que les subventions provinciales et municipales, augmente régulièrement depuis une vingtaine d'années au Québec (QUÉBEC (PROVINCE). MINISTÈRE DE LA CULTURE, 1993; QUÉBEC (PROVINCE). MINISTÈRE DE LA 
CULTURE ET DES COMMUNICATIONS, 1994-2005; QUÉBEC (PROVINCE). MINISTÈRE DE LA CULTURE, DES COMMUNICATIONS ET DE LA CONDITION FÉMININE， 2007; QUÉBEC (PROVINCE). MINISTÈRE DES AFFAIRES CULTURELLES, 1987-1992). Les nouveaux services offerts (liés aux nouveaux supports) sont tarifés (BOISVERT, 1993, p.194). En 1985, 34\% des bibliothèques publiques autonomes tarifiaient l'abonnement; elles étaient $56 \%$ à tarifer pour tous, et $7 \%$ à tarifer selon l'âge en 1999 (LAVOIE, 2000). Généralement la tarification porte sur les abonnements, les pénalités et les locations. Par exemple, la moitié des bibliothèques publiques louent des best-sellers, surtout en vue de réduire les listes d'attentes (BOISVERT, 2000). Lavoie a également montré que l'accès aux ordinateurs était souvent payant (2000). Les débuts de la « guerre contre la gratuité » remonte à 1978 quand le rapport du comité conjoint Québec-municipalités incitait à la taxation des services municipaux pour éviter la «surconsommation » (LYNCH, 1995; PALVADEAU, 1997). Même si la Commission d'études sur les bibliothèques publiques a démontré l'incidence de la tarification sur la baisse de l'utilisation des services de bibliothèques (1987), les élus locaux ont refusé, par l'intermédiaire de l'Union des Municipalités du Québec (UMQ), que le principe de gratuité des bibliothèques soit inscrit dans un texte de loi (PALVADEAU, 1997). De plus, le ministère des Affaires municipales a publié en 1992 un guide intitulé La tarification des services municipaux: document d'information générale, qui donnait aux élus locaux les arguments et les outils pour tarifer leurs services (PALVADEAU, 1997). La tarification est « une question de valeurs sociales et humaines et de priorités professionnelles »(ROUSSEAU, 1993, p.41). La tarification est donc au cœur des débats des bibliothèques publiques québécoises, opposant deux manières de pensée. Les uns voient la tarification comme « un outil de saine gestion » (ROUILLARD, 1993, p.37), et les autres comme une grave entrave aux missions essentielles des bibliothèques publiques (ROUSSEAU, 1993). Ainsi, la tarification des services permettrait de sensibiliser les usagers quant à l'importance des services offerts. Des avis plus nuancés proposent de ne pas tarifer les services de base des bibliothèques mais certains services connexes comme la carte du citoyen, l'abonnement pour les non-résidents, le prêt de documents audiovisuels, les prêts entre bibliothèques, le prêt d'œuvres d'art, les impressions, l'accès aux ordinateurs, les recherches bibliographiques sur bases de données commerciales, locations de salles, etc. (POULIN; STEPHEN, 1995). La tarification est, pour ses défenseurs, la seule solution pour maintenir et améliorer même les services des bibliothèques publiques (ROUILLARD, 1993). Ce point de vue est axé sur le court terme, celui de la balance 
comptable (LAVOIE, 2000). Pour ses détracteurs, la tarification équivaut à considérer l'information comme un bien matériel soumis aux lois du marché (GIAPPICONI, 1993; ROUSSEAU, 1993). Pour Rousseau et Giappiconi, l'information est au contraire un bien public à partager. Les tenants de la gratuité arguent des valeurs essentielles des bibliothèques publiques, et dénoncent la double taxation des personnes _usagers et contribuables à la fois_ (GIAPPICONI, 1993; ROUSSEAU, 1993). Ils craignent que la tarification soit un simple moyen pour éloigner les «indésirables » sans avoir à l'afficher clairement (GIAPPICONI, 1993; LAVOIE, 2000). Ils proposent, pour réaliser des économies dans les bibliothèques publiques et éviter la taxation des services, une meilleure gestion par la coopération et la centralisation pour les acquisitions et le traitement documentaire, le partage des ressources, etc. (GIAPPICONI, 1993; ROUSSEAU, 1993). Ce point de vue est axé sur le long terme, celui de la démocratisation du savoir, de l'éducation permanente. Mais, cette manière de voir est de moins en moins courante au Québec, sans doute parce que les défenseurs de la gratuité ne se sont pas battus sur les bons fronts (les journaux que lisent les élus et non les revues de bibliothéconomie) ni avec les bons arguments (les enjeux sociaux et économiques de l'alphabétisme et de l'éducation permanente et non les valeurs humanistes) (LAVOIE 2000). Quand on sait que le Québec se situe en deçà de la moyenne canadienne en matière d'alphabétisme et quand on connaît le coût de l'analphabétisme, la gratuité des bibliothèques paraît être une nécessité (LAVOIE, 2000). Deux études, entre autres, ont été entreprises en 1987, quand la tarification des services a commencé à inquiéter sérieusement les bibliothécaires. Le rapport de l'ASTED a montré que l'origine de la tarification était reliée à l'apparition des bases de données commerciales, de l'industrie de l'information, des difficultés budgétaires pour les municipalités, de l'augmentation des coûts et de l'accroissement de la demande de services (ROUSSEAU, 1990, p.26). L'ASTED a également montré les conséquences de tarification sur la bibliothèque : baisse de la fréquentation; changement de perception, de comportement et d'attente des usagers; augmentation des frais de gestion; limitation de l'accès à l'information; et augmentation du budget de la bibliothèque dans les cas où la municipalité reporte les bénéfices de la tarification sur le budget de celle-ci (ROUSSEAU, 1990, p.27). Un doute persiste sur ce dernier point (GIAPPICONI, 1993; ROUSSEAU, 1990). Pour Lauzon, les conséquences de la tarification de l'abonnement sont sensiblement les mêmes : iniquité, diminution du nombre d'abonnés et du nombre de prêts et frais de gestion élevés (1995). Boisvert a ainsi démontré que la gratuité engendrait 27\% plus de prêts (1993, p.6). Il existe différentes stratégies tarifaires selon Eymard (2001). Lorsqu'elle 
est orientée par les coûts, la tarification se traduit le plus souvent par un forfait d'abonnement ou un coût par document emprunté. Lorsqu'elle est orientée par la demande, la tarification limite les quantités comme le temps d'accès aux ordinateurs ou le crédit limité d'impression. Il s'agit de limiter la demande, la régulant, la segmentant selon les catégories d'usagers. Lorsqu'elle est orientée vers les objectifs du personnel, la tarification est fonction des coûts que sa gestion génère (procédures, perception, questions des usagers, etc.). Lorsqu'elle est orientée par la concurrence, la tarification a pour but d'éviter une concurrence déloyale. Enfin, lorsque la tarification est orientée vers la tutelle, la tarification est fonction de la mission assignée à la bibliothèque.

La revue de littérature concernant le financement des bibliothèques publiques au Québec démontre l'insuffisance des moyens. Le désengagement financier de l'État est unanimement dénoncé, malgré les trois programmes offerts. L'effort budgétaire des communes et reconnu, mais demeure insuffisant pour donner aux bibliothèques publiques un nouvel élan. Quant à la tarification des services, si elle est dénoncée par les milieux professionnels, elle séduit de plus en plus d'élus locaux. Le financement du développement des bibliothèques publiques au Québec a une incidence néfaste sur les résultats des bibliothèques mais aussi sur l'alphabétisme et l'accès à l'information, comme le montre les différentes études et les comparaisons avec l'Ontario notamment. Quand les subventions portent essentiellement sur les collections, c'est que la priorité est donnée au contenu des bibliothèques et non à la qualité du service rendu par un personnel compétent, complètement absent des incitations financières destinées aux bibliothèques publiques. Le volume du financement, les parts de l'État et des municipalités, la tarification des services ont des incidences directes et rapides sur le développement des bibliothèques publiques, et démontrent l'intérêt que lui portent les élus nationaux et locaux. Si l'argent est le nerf de la guerre, le financement est certainement le facteur de développement des bibliothèques publiques le plus sensible, et ce de deux manières. D'une part, le financement des bibliothèques a une incidence directe et immédiate sur les ressources documentaires, humaines, les espaces, les équipements et les services offerts. D'autre part, le financement est soumis aux aléas économiques très variables d'une année à l'autre et d'une ville à l'autre, et à la volonté des élus nationaux comme locaux. Or, « adequate levels of funding are crucial to the success of a public library in fulfilling its roles

$»$ (INTERNATIONAL FEDERATION OF LIBRARY ASSOCIATIONS AND INSTITUTIONS, 2000, p.14). 


\section{LES NORMES}

Les normes sont des outils de planification, d'organisation et d'évaluation des services pour les gestionnaires des bibliothèques. Ceux-ci peuvent ainsi s'appuyer sur les normes pour justifier leurs demandes budgétaires (COMMISSION D’ÉTUDE SUR LES BIBLIOTHÈQUES PUBLIQUES DU QUÉBEC, 1987). La Commission d'étude sur les bibliothèques publiques du Québec (1987) a démontré que lorsque les normes sont révisées régulièrement, les bibliothèques se développent rapidement.

Pour la Commission d'étude sur les bibliothèques publiques du Québec, la catégorisation des normes est la suivante (1987): (1) les normes peuvent être des normes d'excellence, elles présentent alors un modèle à atteindre; (2) les normes peuvent indiquer des conditions minimales de qualité, elles constituent un seuil en deçà duquel la qualité du service est remise en cause; (3) les normes peuvent être indicatives, elles proposent ce qui serait souhaitable pour assurer la qualité des services; (4) les normes peuvent être impératives, elles constituent une condition sine qua non pour obtenir des aides; (5) les normes peuvent être quantitatives, elles concernent principalement les ressources documentaires, le personnel, les espaces; (6) les normes peuvent être qualitatives, elles mettent l'accent sur l'organisation des services de la bibliothèque; (7) les normes peuvent être générales, elles proposent des orientations; (8) les normes peuvent être précises, elles proposent des critères précis; (9) les normes peuvent être créées par l'autorité gouvernementale, elles sont officielles; (10) les normes peuvent être reconnues par l'autorité gouvernementale, elles sont alors officieuses.

Les premières normes pour les bibliothèques publiques ont été publiées par l'ALA en 1934, lesquelles ont été remises à jour régulièrement (COMMISSION D’ÉTUDE SUR LES BIBLIOTHÈQUES PUBLIQUES DU QUÉBEC, 1987).

Les normes ont fait l'objet d'une homogénéisation, d'une normalisation, notamment par l'UNESCO qui rassemble régulièrement des statistiques internationales depuis 1950 et par l'ISO (GIAPPICONI; CARBONE, 1997).

Depuis les années 1970, l'ALA se bat contre les normes. En 1971, un comité (PUBLIC LIBRARY ASSOCIATION STANDARDS COMMITTEE) proposait une autre manière d'évaluer les services par un processus de planification (planning process) qui s'appuie sur des indicateurs de performance et soit orientée vers l'usager et le service. L'uniformité, l'absence de prise en compte du contexte, l'orientation institutionnelle et le manque de 
rigueur étaient mis en cause dans les normes traditionnelles (COMMISSION D’ÉTUDE SUR LES BIBLIOTHÈQUES PUBLIQUES DU QUÉBEC, 1987). Le Planning Process for Public Libraries publié en 1980 par la Public Library Association explicitait les méthodes de collecte de données et d'interprétation des résultats (GIAPPICONI; CARBONE, 1997). Il a permis aux bibliothèques de se comparer, de mesurer les besoins de la communauté et d'établir des objectifs concrets. (COMMISSION D'ÉTUDE SUR LES BIBLIOTHÈQUES PUBLIQUES DU QUÉBEC, 1987; MAACK). En 1982, les Output Measures for Public Libraries permettaient de mesurer la performance des bibliothèques par rapport aux objectifs fixés et d'établir un diagnostic (COMMISSION D'ÉTUDE SUR LES BIBLIOTHÈQUES PUBLIQUES DU QUÉBEC, 1987; MAACK). Ces outils présentent plusieurs avantages :

\begin{abstract}
une meilleure prise de décision des gestionnaires grâce à de meilleures informations concernant ce qui se passe dans la bibliothèque;une meilleure communication avec les différents paliers de gouvernement et le public concernant les activités de la bibliothèque; une démonstration de la volonté des gestionnaires de rendre leur bibliothèque la plus efficace possible; une meilleure justification concernant l'allocation du budget; et, enfin, une démonstration documentée des améliorations du service (COMMISSION D’ÉTUDE SUR LES BIBLIOTHÈQUES PUBLIQUES DU QUÉBEC, 1987, p.174).
\end{abstract}

Les indicateurs de performance sont de plus en plus considérés dans le monde, en témoignent les communications dans les congrès de l'IFLA et les manuels publiés (GIAPPICONI; CARBONE, 1997). L'ISO a étudié depuis 1991 la faisabilité d'une norme internationale pour homogénéiser la terminologie et les procédures des mesures de performance (GIAPPICONI; CARBONE, 1997). La norme ISO 11620 est parue en avril 1998 (CARBONE, 1998).

Les premières normes au Québec ont été adoptées par le gouvernement en 1974 sous le nom de Normes pour les bibliothèques québécoises. Il s'agissait de normes ni minimales ni d'excellence pour les bibliothèques publiques autonomes. Elles mêlaient des critères qualitatifs et quantitatifs. Elles étaient indicatives et officielles (COMMISSION D’ÉTUDE SUR LES BIBLIOTHÈQUES PUBLIQUES DU QUÉBEC, 1987). Ces normes ont permis, selon la Commission d'étude sur les bibliothèques publiques du Québec (1987), de donner une orientation qui a aidé au développement des bibliothèques publiques au Québec et de leurs services. Ces normes ont été largement utilisées, notamment grâce à leur simplicité (COMMISSION D’ÉTUDE SUR LES BIBLIOTHÈQUES PUBLIQUES DU QUÉBEC, 1987). Mais la Commission a, dans le même temps, dénoncé le manque de révisions (ASSOCIATION POUR L'AVANCEMENT DES SCIENCES ET DES TECHNIQUES DE 
LA DOCUMENTATION, 1996; COMMISSION D’ÉTUDE SUR LES BIBLIOTHÈQUES PUBLIQUES DU QUÉBEC, 1987).

En 1996, l'ASTED a publié Pour des bibliothèques québécoises de qualité : Guide à l'usage des bibliothèques publiques. Elles concernent l'organisation administrative, les changements technologiques et la coopération, les services à la clientèle, le personnel, les collections et les locaux. Les critères quantitatifs, donnés à titre indicatif, concernent les heures d'ouverture par semaine, le nombre d'ordinateurs, le nombre de livres par habitant, le taux de livres pour adultes et pour enfants, le taux d'ouvrages de référence, le nombre d'abonnements aux périodiques, le nombre de mètres carrés, les espaces pour les livres, pour les usagers, pour le personnel, pour les locaux additionnels. Sinon, le guide comporte 49 recommandations (ASSOCIATION POUR L'AVANCEMENT DES SCIENCES ET DES TECHNIQUES DE LA DOCUMENTATION, 1996). Ces normes sont toujours valides.

Les normes sont des outils professionnels qui ont une grande incidence sur le développement des bibliothèques publiques en ce sens qu'elles sont prises en compte par le gouvernement, même si elles sont indicatives et non impératives. Elles permettent d'évaluer et de planifier les services, mais aussi de justifier les attentes vis-à-vis des autorités. Celles-ci reconnaissent les normes et ajustent leurs programmes sur ces dernières; d'où l'importance d'avoir des normes actuelles. Or, le temps écoulé entre deux révisions est de beaucoup trop long au Québec (plus de 10 ans) alors que le contexte des bibliothèques change bien plus rapidement. Cette lenteur est unanimement dénoncée. Une mise à jour des normes est en cours par l'ASTED et est très attendue.

Des normes traditionnelles devraient être maintenues au Québec tant que la situation des bibliothèques publiques sera aussi peu enviable indiquait la Commission d'étude sur les bibliothèques publiques du Québec en 1987. Elle recommandait aussi que les indicateurs de performance soient introduits dans les pratiques professionnelles. Dix-sept ans plus tard, ce premier avis est toujours d'actualité, malheureusement. Quant à l'évaluation de la performance, elle a pénétré les pratiques des professionnels québécois si l'on en croit le nombre de publications sur ce sujet.

\section{CONCLUSION}


L'absence d'une loi spécifique aux bibliothèques publiques est unanimement dénoncée. Aussi, la situation des bibliothèques publiques paraît-elle bien fragile face aux pouvoirs politiques provinciaux et locaux (par exemple lors d'une fusion et/ou d'un démembrement). L'action gouvernementale ne s'inscrit pas dans la durée et le désintéressement de l'État vis-àvis des bibliothèques publiques est remarqué depuis le milieu des années 1980. L'absence d'une direction des bibliothèques publiques, et finalement d'une action concertée et durable nuisent à leur développement.

Les autorités politiques québécoises ont toujours privilégié la gestion municipale des bibliothèques publiques. L'option des conseils d'administration, courante chez nos voisins anglo-saxons, a fait preuve de son efficacité. Elle permettrait notamment de soustraire le développement des bibliothèques publiques de l'arène politique, par définition instable dans une démocratie. Elle placerait du même coup la bibliothèque au cœur de la communauté.

L'insuffisance des aides financières gouvernementales est dénoncée dans la littérature, ainsi que l'accent mis sur l'accroissement des collections au détriment des ressources humaines notamment. Si l'importance de la part de financement assurée par les municipalités est reconnue, l'augmentation des revenus issus de la tarification des services inquiète.

Une mise à jour régulière des normes s'impose étant donné la rapidité des bouleversements, notamment technologiques, que connaissent les bibliothèques publiques.

Les facteurs de développement des bibliothèques publiques sont pluriels. Ils inter-agissent. C'est précisément cet enchevêtrement de facteurs explicatifs qui font tout l'intérêt de la recherche dans ce domaine.

\section{BIBLIOGRAPHIE}

ASSOCIATION POUR L'AVANCEMENT DES SCIENCES ET DES TECHNIQUES DE LA DOCUMENTACION (ASTED). Des bibliothèques pour un meilleur développement culturel au Québec. Documentation et bibliothèques, v. 37, n. 4, p.133-35, 1991.

Pour des bibliothèques québécoises de qualité: guide à l'usage des bibliothèques publiques. Montréal: ASTED, 1996.

La bibliothèque publique. In: Donnons-nous la chance de réussir: capitaliser sur 
nos expertises en travaillant ensemble! Montréal: ASTED, 1998.

BAILLARGEON, J. P. Les bibliothèques publiques du Québec, 1961-1989. Documentation et bibliothèques, v. 38, n. 3, p. 139-47, 1992.

BOISVERT, D. La tarification, qui en profite? Défi, p. 15, 1992.

- La spécificité des bibliothèques publiques du Québec. Documentation et bibliothèques, v. 39, n. 4, 191-96, 1993.

La tarification. Défi, p. 6-9, 1993.

La location de best-sellers dans les bibliothèques publiques du Québec contribue-telle vraiment à affaiblir la chaîne du livre? Defi, v. 2, n. 2, 2000. En ligne : <http://www.bpq.org/RevueDEFI/2-2/President.html>. Consulté en 27 abr. 2004.

CARBONE, P. Évaluer la performance des bibliothèques. Bulletin des bibliothèques de France, v. 43, n. 6, p. 40-45, 1998.

CHAREST, C. Les revenus des bibliothèques publiques: subventions en perte de vitesse. Défi, p. 14-18, 1995.

CHRISTENSON, J. Role of the Public Library Trustee. Library Trends, v. 44, n. 1, p. 63 $76,1995$.

COMMISSION D’ÉTUDE SUR LES BIBLIOTHÈQUES PUBLIQUES DU QUÉBEC. Les bibliothèques publiques: une responsabilité à partager. [s.l.] : Commission d'étude sur les bibliothèques publiques du Québec, 1987.

CRÉPEAU, I. La législation des bibliothèques publiques du Québec à travers l'histoire: essai de synthèse. Documentation et bibliothèques, v. 45, n. 1, p. 25-33, 1993.

EYMARD, D. Éléments de stratégies tarifaires. Bulletin des bibliothèques de France, v. 46, n. 6 , p. 68-72, 2001.

GAGNON, G. La politique d'aide au développement des bibliothèques publiques du Québec (1960-1985). Documentation et bibliothèques, v. 31, n. 1, p, 9-25, 1985. 
GAMACHE, A. La tarification dans les bibliothèques: une autre expérience éloquente. Défi, p. 14, 1992.

GARDNER, F. Législation relative aux bibliothèques publiques: étude comparative. Paris: UNESCO, 1972.

GIAPPICONI, T. La tarification et ses masques. Bulletin des bibliothèques de France, v. 38, n. 2, p. 10-22, 1993.

GIAPPICONI, T.; CARBONE, P. Évaluer et contrôler. Management des bibliothèques: programmer, organiser, conduire et évaluer la politique documentaire et les services des bibliothèques de service public. Paris: Le cercle de la librairie, p. 225-56, 1997.

HARRIS, M. H. Public Libraries and the Decline of the Democratic Dogma. Library Journal, v. 101, n. 19, p. 2225-30, 1976.

HERNON, P.; ATMAN, E. Assessing Service Quality: Satisfying the Expectations of Library Customers. Chicago: American Library Association, 1998.

INTERNATIONAL FEDERATION OF LIBRARY ASSOCIATIONS AND INSTITUTIONS. Revision of IFLA's Guidelines for Public Libraries. En ligne: $<$ http://www.ifla.org/VII/s8/proj/gpl.htm>. Consulté en 2000.

LABORIT, L.; DUBOIS, F. Les bibliothèques publiques trois ans après le Plan Vaugeois. Argus, v. 12, n. 3-4, p. 69-73, 1983.

LAMONDE, Y. Une contribution à l'histoire de la bibliothèque publique au XIXe siècle. In : Les bbliothèques québécoises d'hier à aujourd'hui: actes du colloque de l'ASTED et de l'AQUEI, Trois-Rivières.. Association pour l'avancement des sciences et des techniques de la documentation (ASTED). Montréal: ASTED, n. 27, p.21-27, out., 1997.

LAUZON, G. Conséquences de la tarification de l'abonnement. Défi, p. 37-38, 1995.

LAVOIE, S. Tarification jeunesse dans les bibliothèques publiques du Québec: ticket modérateur vers l'alphabétisme? Défi, v. 2, n. 1, 2000. En ligne : $<$ http://www.bpq.org/RevueDEFI/2-1/tarification.html>. Consulté en 27 abr. 2004.

LEGAULT, S. Fusions municipales et enjeux pour les bibliothèques publiques. Documentation et bibliothèques, v. 47, n. 2, p. 53-60, 2001. 
LYNCH, J. M. La tarification des services municipaux et la bibliothèque publique. Défi, p. 32-34, 2005.

MALONE, C. K. Toward a Multicultural American Public Library History. Libraries \& Culture, v.35, n. 1, p. 77-87, 2000.

MATTE, P. Bibliothèques publiques et conseils d'administration. Documentation et bibliothèques, v. 33, n. 2, p. 45-49, 1987.

MITTERMEYER, D. Le conseil d'administration des bibliothèques publiques: sa composition. Défi, v. 2, n. 1, p. 7, 1986. p.7-8, 1986.

Le conseil d'administration des bibliothèques publiques: ses origines. Défi, v. 1, n. 3, Public Libraries: Boards Don't Make a Difference. Canadian Library Journal, v. 43, p. 434-35, 1986.

Le conseil d'administration des bibliothèques publiques: ses avantages et ses inconvénients. Défi, v. 2, n. 2, p.7, 1987.

- Le conseil d'administration des bibliothèques publiques: ses avantages et ses inconvénients (2 Parte). Défi, v. 2, n. 3, p 8-9, 1987.

Le développement des bibliothèques publiques du Québec: un pas en avant, deux pas en arrière. Argus, v. 17, n. 2, p. 37-46, 1988.

. The Public Library Boards of Trustees Versus the Committees of City Council: a power game. Canadian Journal of Information and Library Science, v. 19, n. 1, p. 1-17, 1994.

Rendement des bibliothèques publiques: une analyse des valeurs, des intérêts, et des bénéfices. Revue canadienne des sciences de l'information et de bibliothéconomie, v. 24, n. 4, p. 1-32, 1999.

MORIN, D.; LAJEUNESSE, M. Les bibliothèques publiques du Québec 1977-1992: analyse comparée des performances des bibliothèques autonomes. Argus, v. 25, n. 1, p. 14-27,1996. 
PALVADEAU, C. Gratuité ou tarification dans les bibliothèques publiques québécoises. Bulletin d'informations de l'Association des bibliothécaires français, v. 174, n. 1, p. 183 85, 1997.

PANNETON, J. La lecture publique au Québec de 1959 à demain. Documentation et bibliothèques, v. 41, n. 3, p. 153-58, 1995.

POULAIN, M. Retourner à Tocqueville pour comprendre l'histoire comparée des bibliothèques américaines et françaises au XIXe siècle. Bulletin des bibliothèques de France, v. 47, n. 5, p. 66-73, 2002.

POULIN, C.; STEPHEN, M. T. La tarification des bibliothèques: une question de nuances. Défi, p. 35-36, 1995.

QUÉBEC (PROVINCE). Le temps de lire, un art de vivre: politique de la lecture et du livre. Québec: Ministère de la culture et des communications, 1998.

Ministère de la culture. Bibliothèques publiques du Québec: statistiques. Québec: Ministère de la culture, 1993.

Ministère de la culture et des communications. Bibliothèques publiques du Québec: statistiques. Québec : Ministère de la culture et des communications, 1994-2001.

Bibliothèques publiques du Québec: statistiques. Québec: Ministère de la culture et des communications, 2002.

Bibliothèques publiques du Québec: statistiques 2003-2005. En ligne : $<$ http://www.mcccf.gouv.qc.ca/fileadmin/documents/publications/stat-bibliotheques-publ2003.pdf $>$; $\quad<$ http://www.mcccf.gouv.qc.ca/fileadmin/documents/publications/statbibliotheques-publ-2004.pdf $>$;

$<$ http://www.mcccf.gouv.qc.ca/fileadmin/documents/publications/stat-bibliotheques-publ2005.pdf>. Consulté en 29 abr 2004.

Ministère de la Culture, des Communications et de la Condition féminine. 2008. Bibliothèques publiques : Statistiques 2006. [en ligne]: $<$ http://www.mcccf.gouv.qc.ca/fileadmin/documents/publications/stat-bibliotheques-publ2006.pdf>. Consulté en 29 abr 2004.

Ministère de la culture et des communications. Loi sur le Ministère de la culture et des communications. L.R.Q., chapitre M-17. 2004. En ligne : Enc. Bibli: R. Eletr. Bibliotecon. Ci. Inf., ISSN 1518-2924, Florianópolis n. esp., 1. sem. 2009. 
$<$ http://www2.publicationsduquebec.gouv.qc.ca/dynamicSearch/telecharge.php?type=2\&file= /M_17_1/M17_1.HTM>. Consulté en 29 abr. 2004.

Ministère des affaires culturelles. Bibliothèques publiques du Québec: statistiques. Québec: Ministère des affaires culturelles, 1987 - 1992.

RIGOGNE, A. H.; RÉJEAN, S. Bibliothèques publiques du Québec: panorama sur les services offerts. Bulletin des bibliothèques de France, v. 39, n. 4, p. 46-54, 1994.

ROUILLARD, C. La tarification des services documentaires: un outil de saine gestion. Documentation et bibliothèques, v. 39, n. 1, p. 37-38. 1993.

ROUSSEAU, D. La tarification des services documentaires: de la théorie à la pratique: compte-rendu de deux études. Documentation et bibliothèques, v. 36, n. 1, p. 25-33, 1990.

1993.

. La gratuité? Et comment! Documentation et bibliothèques, v. 39, n. 1, p. 38-41,

SÉGUIN, F. Bibliothèques publiques québécoises: radioscopie d'un retard. Défi, p. 8-12, 1995.

Fusion des villes de Montréal: quelques interrogations sur l'avenir des bibliothèques Municipales." Défi, v. 3, n. 1, 2001. En ligne : <http://bpq.org/3-1/seguin.html>. Consulté en 29 abr. 2004.

SIMON, M. L. Les bibliothèques publiques du Québec: un échec. Argus, v. 23, n. 1, p. 4-5, 1994.

UNITED NATIONS EDUCACIONAL SCIENTIFIC AND CULTURAL ORGANIZATION - (UNESCO). Manifeste de l'UNESCO sur la bibliothèque publique. En ligne : $<$ http://www.unesco.org/webworld/libraries/manifestos/libraman_fr.html.> Consulté en 1994.

VALLET, C. En direct du Québec: bilan et perspectives pour les bibliothèques publiques. Documentation et bibliothèques, v. 32, n. 6, p. 502-523, 1987.

VAN HOUSE, N. A.; CHILDERS, T. A. The Public Library Effectiveness Study: the Complete Report. Chicago: American Library Association, 1993. 


\title{
Resumo
}

Este artigo é uma revisão de literatura que propõe distinguir os diferentes fatores de desenvolvimento das bibliotecas públicas quebequenses. A legislação, a ação governamental, os estilos administrativos, o financiamento e as normas são estudados a fim de apresentar os processos de desenvolvimento das bibliotecas públicas no Quebec.

Palavras-chave: Bibliotecas públicas - Quebec. Ação governamental. Estilos administrativos, financiamento e normas.

\begin{abstract}
This article is a literature review which proposes to distinguish different factors in the development of public libraries in Québec. Legislation, governmental support, administrative modes of management, funding and standards are studied to report the process of development of public libraries in Québec.
\end{abstract}

Keywords: Public libraries - Québec. Governmental support, Administrative modes of management, funding and standards.

Originais recebidos em: 05 de março de 2009

Aceitos para publicação em: 14 de maio de 2009 